\title{
Wider das Gemurmel oder wieder das Gemurmel?
}

\section{Von Thomas Hinz}

Freitag, der 13. Oktober. In der Universität Kassel geht der Soziologiekongress zu Ende, in Bonn geben DFG und Wissenschaftsrat zur selben Zeit die Entscheidungen zur ersten Förderrunde der Exzellenzinitiative bekannt. Die in Kassel bis zum Schluss ausharrenden Teilnehmer hören die Diskussion mit dem Göttinger Neurobiologen Gerald Hüther, der auch als Autor populärwissenschaftlicher Bücher mit Titeln wie »Bedienungsanleitung für ein menschliches Gehirn« oder »Die Evolution der Liebe« bekannt wurde. Durch dieses zeitliche Zusammentreffen lädt das Kongressthema »Die Natur der Gesellschaft« ganz besonders dazu ein, das Verhältnis der Soziologie als Sozialwissenschaft gegenüber den von KarlSiegbert Rehberg (Dresden) in seiner Eröffnungsrede als dominant herausgestellten Naturwissenschaften auszuloten. Keine Woche vergeht ohne neue naturwissenschaftliche Erkenntnisse - eher selten sind sie angesichts des schon bekannten Wissensbestands wirklich bahnbrechend, oftmals mit für die Öffentlichkeit schwer verständlichen, aber doch schwerwiegenden methodischen Problemen behaftet und, wie wir wissen, manchmal sogar gefälscht. Trotzdem wird das, was die »Gesellschaft« als Wissenschaft begreift, immer mehr durch die Naturwissenschaften geprägt, ein Umstand, der in der Programmförderung zu Gunsten von angewandten Naturwissenschaften zum Ausdruck kommt und in den Entscheidungen vom 13. Oktober zur Zukunft der Forschungslandschaft an deutschen Hochschulen nicht weniger.

In dieser Situation den Blick auf die Natur der Gesellschaft zu richten, ist aller Ehren wert, vielleicht sogar mutig und - angesichts des Zwangs zur Selbstbehauptung der Soziologie an vielen Universitäten - auf jeden Fall überfällig im Hinblick auf die eigene Disziplin, aber mehr noch bezüglich des Verständnisses von Gesellschaft und der Erklärung sozialer Prozesse. Es liegt auf der Hand, prominente Themen der Naturwissenschaften in der Umweltforschung (Klimawandel), der Demographie (Vergreisung, Kinderlosigkeit) oder der Biologie (Beginn und Ende des Lebens) mit der Soziologie zu verknüpfen. Den Brückenschlag fordert die Sache selbst. Die Ingenieurwissenschaften wissen es auch schon: Die Natur wurde, jedenfalls solange es den homo faber gab, immer »gemacht « und dabei eingesetzte Techniken hatten und haben enorme gesellschaftliche Auswirkungen. Werner Rammert (Berlin) stellte in seinem Plenarbeitrag zum Kongressthema klar: Feldmaus und Hausmaus sind biologische wie soziale Tatsachen. Eine nicht ganz neue Einsicht der Anthropologie sollte den Sozialwissenschaften ohnehin den Rücken stärken: Der Mensch ist qua Natur ein instinktarmes Sozial- und Kulturwesen. Trotz aller epochaler Entdeckungen der Natur- und - wie man inzwischen differenziert - Lebenswissenschaften bleiben viele Fragen für die Soziologie jedoch im besten Sinne offen: Welche konkreten Formen nimmt Sozialität in der Mikro- und Makroperspektive an? Welche Rolle spielt dabei die Entwicklung von Kultur und Institutionen? Wie wird Natur als Ressource eingesetzt? Welche Vorstellungen von Natur machen sich Menschen und was folgt daraus für die Existenz einzelner Menschen, ganzer Gesellschaften, ja der Weltgesellschaft, um nur einige nahe liegende Forschungsfragen aufzuzählen. Somit wäre also für jede Menge Gesprächs- und Diskussionsstoff gesorgt gewesen, der für die Kongressbesucher und die Beitragenden von Interesse sein sollte. Man kann natürlich einwenden, die Erwartungen an das zweijährlich stattfindende Ereignis eines Soziologiekongresses seien damit mächtig überzogen, schließlich ist eine solche Veranstaltung in erster Linie eine willkommene Gelegenheit zum Wiedersehen, eine »Inklusionsparty«, wie Jürgen Kaube in der Frankfurter Allgemeinen schrieb. Schon richtig, dennoch soll versucht werden, die präsentierten Antwortvorschläge auf die angesprochenen Fragen

Soziale Welt 58 (2007), S. $101-105$ 
zumindest ein wenig zu sortieren. Schafft es die Soziologie angesichts einer weit entwickelten disziplinären Differenzierung von Theorien und Methoden noch über ein gemeinsames und selbst gesetztes Thema zu diskutieren? Kann die Soziologie angesichts der wahrgenommenen Dominanz der Naturwissenschaften gute Argumente für ihre eigene wissenschaftliche Existenz und ihre Mitwirkung an der Lösung der aufgeworfenen Fragen entwickeln und kommunizieren? Oder gleicht unsere Kommunikation mit der eigenen Zunft und der breiteren Öffentlichkeit eher einem selbstbezogenen und in schlimmeren Fällen selbstgefälligen Gemurmel?

Die Soziologie entstand als eigenständig wahrgenommene Wissenschaft in deutlicher Abgrenzung zur Biologie, interessanterweise mit erheblichen Anleihen, was die Argumentationsfiguren angeht. Im Selbstverständnis vieler Soziologen spielt die schroffe Zurückweisung, man kann sagen die Demaskierung »biologistischer« Erklärungen eine zentrale Rolle. In der Gender Debatte wirkt der Gründungsmythos der Wissenschaft von den »sozialen Tatsachen « etwa dahingehend, die Ergebnisse biologischer und psychologischer Forschung vornehmlich als Material für eine Dekonstruktion heranzuziehen. Dabei ist das, was die Lebenswissenschaften zur Entstehung des (individuellen) Wahrnehmens, Wissens und Fühlens beitragen (ob bei Frauen oder Männern), auch substanziell von Belang. Auch die Diskussion über kognitive Fähigkeiten ist immer noch nicht frei von der verfehlten Gegenüberstellung von nature und nurture. In der Soziologie herrschen hier die gewohnt ideologiekritischen Töne vor, die unbestreitbar nötig sind, wenn etwa Bildungsdefizite zu einem wichtigen Teil durch Institutionen produziert werden. Solche Analysen können einer Politik, die vor allem mit einer natürlichen Vererbung von Ungleichheiten argumentiert, entgegenwirken, sie bringen aber die Suche nach neuen wissenschaftlichen Erkenntnissen kaum weiter. Die überkommene Grenzziehung zwischen Soziologie und Biologie nahm Renate Mayntz (Köln) zum Anlass eines von ihr veranstalteten »Schattenboxens«. Renate Mayntz wurde zur Kongresseröffnung mit dem diesjährigen Preis der DGS für das soziologische Lebenswerk ausgezeichnet und war von dieser Ehrung offenbar auch selbst ein wenig überrascht. Ihre sozialwissenschaftlichen Arbeiten waren Anregungen und Impulsen aus anderen Disziplinen nie verschlossen und so führte sie aus der Perspektive einer interdisziplinär ausgewiesenen, erfahrenen und erfolgreichen Forschungsarchitektin die imaginären Boxer in den Ring. Die Soziologie, so Mayntz, muss sich nicht in der Ringecke verstecken. Die Thesen der Neurowissenschaften zum »freien Willen« und zur Selbstverantwortung der Menschen sind sicher ethisch und philosophisch eine Herausforderung, aber für eine Soziologie, die mittels sozialer Mechanismen soziale Prozesse erklären möchte, keinesfalls bedrohlich. Das Gehirn (und damit Kognition, Gefühle etc.) werde erst im Laufe sozialer Erfahrungen »programmiert«. Wie der nach Kassel eingeladene Gehirnforscher Hüther ausführt, beeinflussen soziale Beziehungen, vor allem in frühen Lebensphasen, die Verschaltungen im Gehirn - und dies mit wichtigen Folgen. Im Sinne von Mayntz muss die Soziologie sicher auch die Erkenntnisse der Neuroökonomie zur Bedeutung von Hormonen wie Oxytocin und einer »programmierten« Reziprozität zur Kenntnis nehmen. Der Erklärungsauftrag an die Soziologie, nämlich die sozialen Umstände zu bestimmen, unter denen bestimmte handlungsrelevante Orientierungen oder gar Gefühle aktiviert werden, bliebe weiterhin auf der Agenda, so Mayntz. Für die Entwicklung von Modellen sozialer Prozesse sei eine einfache Handlungstheorie noch immer die beste Grundlage.

Empirische Umsetzungen einer solchen Forschungsprogrammatik waren im Kongressprogramm durchaus zu entdecken, etwa bei Axel Franzens und Sonja Pointners (Aachen) in der Sektion »Modellbildung und Simulation« präsentierten Studie zu der in spieltheoretischen Experimenten geprüften Norm von Reziprozität. Sehr elegant konnte Markus Gangl (Mannheim) im Plenum zur »Naturalisierung von Erziehung, Bildung und sozialer Ungleichheit« zeigen, dass egalitäre Bildungspolitik dann besonders erfolgreich ist, wenn der Zusammen- 
hang von Persönlichkeitseigenschaften (worunter Gangl auch »natürliche«, angeborene Dispositionen fasst) mit späterem Arbeitsmarkterfolg hoch und der Zusammenhang dieser Eigenschaften mit den klassischen in der Bildungsforschung betrachteten sozio-ökonomischen Ressourcen gering ausfällt. Der Beitrag von Gangl steht nicht nur für gute, sondern auch für bildungspolitisch relevante Forschung - und das, obwohl manchen Soziologen verdächtige Größen wie »Talent« im Spiel sind. Der von Mayntz vorgetragene Anspruch an ein soziologisches Erklärungsprogramm wurde ansonsten kaum eingelöst, es überwogen diskurs- und metatheoretische Auseinandersetzungen mit den Erkenntnissen der Naturwissenschaften.

In den letzten Jahrzehnten hat sich das Forschungsinstrumentarium der Soziologie erheblich ausdifferenziert, wobei die Weiterentwicklung von statistischen Techniken und die Erhebung von zeitbezogenen Daten ganz wesentlich beteiligt waren. Dieser Fortschritt ist erst aus einem größeren Abstand erkennbar, wenn man das methodische Niveau vieler Präsentationen mit solchen auf Soziologiekongressen noch Anfang der 1990er Jahre vergleicht. Beim Einsatz von komplexeren und besser an sozialwissenschaftliche Hypothesen angepassten Modellen gibt es auch unmittelbare Verbindungen zu den Natur- und Lebenswissenschaften. Aus dem Programm des Kongresses sind beispielsweise die Arbeiten zu sozialen Netzwerken hervorzuheben, die in einer Plenarveranstaltung vorgestellt wurden und in ihrem methodologischen Zuschnitt auch für Anwendungen in den Naturwissenschaften taugen. In diesem Forschungsgebiet können auch Simulationstechniken gewinnbringend eingesetzt werden, wie Andreas Flache und Tom Snijders (Groningen) gezeigt haben. Die methodische Differenz zwischen den Disziplinen bleibt groß, wenn es um die Interpretation von Sinn geht, der mit Handlungen und Symbolen, ganz allgemein mit Kultur verbunden ist. Wissenssoziologische Studien verweisen auf die sozialen und kulturellen Deutungsprozesse in den »harten« Wissenschaften. Die Forscher dieser Richtung sind selbst und auch selbstbewusst an der Schöpfung von Sinn beteiligt. Aber auch die quantitativen Methoden sind für kulturell geprägte Bilder offen. So argumentierten Ulrich Rosar und Markus Klein (Köln), dass Evaluationsergebnisse in Lehrveranstaltungen durch Einschätzungen von körperlicher Attraktivität der Dozenten beeinflusst werden. Beauty pays - auch im Wissenschaftsbetrieb. Beruhigend ist dagegen die Erkenntnis, dass angesichts der Bedeutung, die frühe Lebensphasen für die menschliche Entwicklung und die Ausprägung sozialer Ungleichheiten haben, Standardinstrumente der Umfrageforschung auch bei Kindern prinzipiell einsetzbar sind (Marek Fuchs, Kassel). Der Blick auf die Methodensektionen belegt, dass der Trend, in den Leitorganen unseres Fachs wie der Zeitschrift für Soziologie, der Kölner Zeitschrift oder der Sozialen Welt fast nur noch Aufsätze von »jungen Leuten« abzudrucken, nicht nur auf die Erfordernisse wissenschaftlicher Karrieren zurückzuführen ist. Alles in allem spricht das methodische Niveau in den Arbeiten des Nachwuchses für ihn. Es ist oft elaborierter als bei den »alten Meistern«. Jene konnten sich auf dem Kongress ganz den großen Themen von Natur und Gesellschaft widmen.

Die Beschäftigung der Soziologie mit der Natur der Gesellschaft wirft auch die Frage nach Gewalt und ihrer sozialen Lösung auf. Bei Thomas Hobbes ist die Natur asozial, sie ist roh und durch Gewalt, den Krieg aller gegen alle gekennzeichnet. Alle Befriedung und alle Zivilisation entsteht durch die Überwindung des Naturzustands, womit der moderne Staat geboren wird. Er monopolisiert die Gewaltausübung, die Menschen werden durch Außen- und Innenkontrollen gezähmt. Jan-Philipp Reemtsma (Hamburg) erklärte in seinem Eröffnungsvortrag die Enttäuschung über das Versprechen einer Soziologie der Gewalt, wie sie das Sonderheft der Kölner Zeitschrift von 1997 verhieß, damit, dass genau die Argumentationsfigur der Befriedung durch das staatliche Gewaltmonopol durch die Geschichte insbesondere des 20. Jahrhunderts widerlegt werde. In einigen Phasen der Geschichte war gerade das Gewaltmonopol des Staates eine Quelle für grausame Gewaltanwendung, wodurch die »Na- 
turhaftigkeit« von Gewalt in Frage steht. Auch bei Anerkennung von genetischen Dispositionen sind wir Menschen von Natur aus nicht gewalttätig oder friedfertig. Gewalt entsteht in sozialen Beziehungen und wird zum sozialen Phänomen, das häufig - aber nicht immer mit dem Zerfall von Sozialität einhergeht. Die auch heute noch aktuelle Drohung der Asozialität durch Gewalt verweist auch auf die »Naturgewalt«. Die Natur als Donnergroll der Götter wird im Zuge der Rationalisierung und Säkularisierung »sinnentleert « - so Bernhard Giesen (Konstanz). Die Entzauberung der (Um-)Welt vollzieht sich im Zuge der Weiterentwicklung der Naturwissenschaften in allen Lebensbereichen. Wir wissen nun um die Ursachen des Donnergrolls und der sich anbahnenden Klimakatastrophe - und Fragen nach dem »Sinn des Lebens « werden allenfalls bei Naturkatastrophen wie einem Tsunami aktuell. Exemplarisch standen dann im Plenarvortrag von Giesen der Müll und seine mögliche Transformation in nützliche oder heilige Dinge im Mittelpunkt. Müll ist das Ergebnis menschlicher Naturaneignung und Konsumentscheidungen. Beide sind angesichts der »menschlichen Natur « kulturell geprägt. Müll stinkt vor allem, er entwertet die Vergangenheit, kann aber auch neuen Sinn entfalten, als Wertstoff oder sogar Kunst. Als weiteres Beispiel diente die Transformation von profanen Stoffresten oder von Überresten des menschlichen Körpers in Reliquien der katholischen Kirche. Eine solche narrativ betriebene Soziologie, wie sie etwa auch im Plenarbeitrag von Klaus Eder (Berlin) zur Naturalisierung von Gesellschaft am Beispiel des Problembären Bruno betrieben wurde, ist zweifelsohne manchmal ein rechtes Vergnügen. Oft bleibt allerdings ein ungewisses Gefühl zurück, dass die beschriebenen Phänomene vielleicht auch ganz anders zu erklären wären.

Über die Natur der Gesellschaft zu sprechen, heißt auch, deren Reproduktion zu durchdenken. Sie vollzieht sich in demographischen Prozessen, Fertilität und Mortalität, Morbidität und Migration. Karl Ulrich Mayer (Yale) verwies darauf, wie lange in der Diskussion um die sexuelle Reproduktion von Gesellschaften der Zwangscharakter von »Bevölkerung als Schicksal « im Hinblick auf gesellschaftliche Entwicklung vorherrschte. Dabei sind die Zusammenhänge zwischen individueller und kollektiver Reproduktion weitaus komplexer und weniger schicksalhaft als sie erscheinen. Die Fertilitätsrate in Deutschland ist, anders als in der öffentlichen Wahrnehmung, nicht erst seit ein paar Jahren niedrig, sie ist für Westdeutschland bereits seit Mitte der 1970er Jahre annähernd unverändert, während andere entwickelte Länder teils noch geringere (wie die osteuropäischen Staaten), teils auch höhere Raten (wie die USA) aufweisen. Interessant ist auch, wie Fertilitätsrate und politische Orientierung korrelieren: In den USA könne der Unterschied dieser Rate zwischen den Bundesstaaten (der baby gap) auf der Makroebene ziemlich perfekt die Unterschiede bei den Stimmenanteilen der Bush-Wähler 2004 vorhersagen. Den Zusammenhang zwischen Demographie und Gesellschaft müsse man, so Mayer, in dynamische Modelle fassen, welche institutionelle, kulturelle und ökonomische Faktoren beinhalten. Wie sehr gesellschaftspolitische Vorstellungen die Reproduktion beeinflussen können, konnten einige Vorträge aus dem und zum Gastland des Kongresses veranschaulichen. Mit starken regionalen Schwankungen wurde in China die Ein-Kind-Familie durchgesetzt - mit einer bekannten Nebenfolge: Das Geschlechterverhältnis hat sich bei chinesischen Kleinkindern im Zuge dieser Familienpolitik und der pränatalen Diagnosemöglichkeiten zur Bestimmung des Geschlechts deutlich verändert, wie Bettina Gransow (Berlin) ausführte. Die missing girls (auf 120 Jungen kommen etwa 100 Mädchen) werden sich absehbar auf verschiedene Aspekte der chinesische Sozialstruktur auswirken, wenn die entsprechenden Geburtskohorten selbst Familien gründen und in den Arbeitsmarkt eintreten. In der Sektion Familiensoziologie gab es weitere Argumente dafür, dass die Natur der sexuellen Reproduktion von sozialen Gelegenheiten abhängt. Partnerwahl sei eben nicht biogenetisch determiniert, sondern setzt bei Möglichkeiten, Partner zu treffen (wie sie etwa durch professionelle Kontaktagenturen im Internet erheblich erweitert werden), bei kulturell konstruierten Vorstellungen von Liebe und bei Verhandlungsspielräumen in Beziehungen an (Stephan Stengel, Mainz). Auch die Vorstellung, die Natur der 
Reproduktion sei auf der Mikroebene technisch beherrschbar, wird angesichts eines Anteils von 30\% ungeplanter Schwangerschaften erschüttert (Michael Feldhaus und Mandy Boehnke, Bremen).

Und sonst? Es ging in Kassel noch um wesentlich mehr, beispielsweise um die hergestellte Natur im Wald, um »Umweltgerechtigkeit « und »Strategien der Biopolitik « sowie wahrlich letzte Dinge wie Sterben und Tod. Der Gesamteindruck war: Die Soziologie kann die Dominanz der Naturwissenschaften erstaunlich gut verkraften, die menschlichen Vorstellungen von Natur haben »Leerstellen« und mit jedem Wissensfortschritt entstehen neue Deutungsmöglichkeiten. Fraglich ist allerdings, ob die vorhandenen und mit dem Kongressthema angesprochenen Möglichkeiten einer Integration von Natur- und Sozialwissenschaften, wie sie etwa in den Büchern des nach Kassel eingeladenen, aber nicht gekommenen Jared Diamond aufscheinen, in der deutschen Soziologie ergriffen werden.

Als Kongressbesucher und Mitglied der Deutschen Gesellschaft für Soziologie bleiben noch einige Anmerkungen. Heinz Bude (Kassel) stellte am Schluss des hier mehrfach erwähnten und dann auch in der Tagespresse besprochenen Hauptplenums fest: »Es macht keinen Sinn mehr zu diskutieren.« Das nicht nur bei diesem Plenum festzuhaltende Fehlen der Diskussion, welches durch organisatorische Vorgaben, wie ein übervolles Programm oder zu lange und abgelesene, manchmal auch gemurmelte Vorträge, regelrecht provoziert wird, ist wohl für viele Kongressteilnehmer demotivierend. Es hat sicher auch positive Erfahrungen gegeben, wie etwa nach Stefan Hirschauers Vortrag »Körper Macht Wissen«. Doch wenn der Trend einer Diskussionsmüdigkeit zuträfe, dann sind die inszenierten und eingeladenen Diskussionen umso wichtiger, etwa author meets critics. Diese Veranstaltungsform ist ein belebendes Element, was man für manche andere vom Vorstand der DGS organisierte Sonderveranstaltung nicht sagen kann. Ein Negativerlebnis war das Forum zur Hochschulpolitik, bei der nicht die soziologische Auseinandersetzung mit paradoxen Folgen der Studiengangreformen anstand, sondern das langatmige Ausbreiten von ungeprüften und auch unprüfbaren Behauptungen. Gegen solche Verschwörungstheorien erscheint die Soziologie zwar inzwischen weitgehend immun wie gegen Ad-Hoc-Erklärungen, die Kongressveranstalter aber offenbar nicht gegen eine extreme Vermehrung von Ad-Hoc-Gruppen. Es gilt das verbandspolitisch nachvollziehbare Argument, wonach die Organisatoren solcher Initiativen der DGS beitreten und die Vortragenden die Teilnahmegebühr bezahlen müssen. Für den einfachen Besucher ist die Konkurrenz sehr vieler Veranstaltungen am Nachmittag und das vergleichsweise magere Angebot am Vormittag ärgerlich - außerdem führt eine ungezügelte Ausweitung der Ad-Hoc-Angebote immer mehr in Richtung »Inklusionsparty«. Man kann dies bewusst betreiben, nur steht dann nicht die Ausstrahlung der Soziologie in der Öffentlichkeit im Vordergrund und auch nicht die Orientierung an den Kongressthemen. So war das Medienecho auf den Soziologiekongress gemessen am zwei Wochen zuvor stattfindenden Historikertag eher gering. Die Öffentlichkeit, vor allem die Leute, die diese Öffentlichkeit herstellen, fordern auch mehr Zuspitzung statt Beliebigkeit. Das größte Medienecho hatte bezeichnenderweise die Kurznotiz, wonach die Höhe des Taschengeldes für die Vorhersage des Bildungserfolgs bei Kindern wichtiger ist als ein Intelligenztest. Das kann nicht alles sein. Die Soziologie ist als Wissenschaftsdisziplin auf eine professionellere Kommunikation mit der Öffentlichkeit angewiesen - und zwar nicht nur im Feuilleton, sondern auf den Wissenschaftsseiten. Wir haben etwas zu sagen: Wider das Gemurmel.

Prof. Dr. Thomas Hinz

Fachbereich Soziologie

Universität Konstanz

Universitätsstr. 10

78457 Konstanz

e-mail: thomas.hinz@uni-konstanz.de 